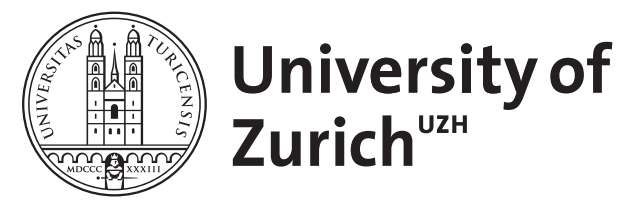

\title{
Psychotherapie von Kriegs- und Folteropfern
}

Maier, T

\begin{abstract}
Zusammenfassung: Im Zuge weltweiter Migrationsbewegungen kommen zunehmend Menschen zu uns, die durch Krieg-, Folter und Vertreibung traumatisiert sind. Die psychotherapeutische Versorgung dieser Menschen ist aus vielen Gründen hindernisreich und deshalb oft unzureichend. Neben eigentlichen psychischen Beschwerden liegen meist zusätzliche körperliche, soziale und aufenthaltsrechtliche Probleme vor. Die Sprachbarriere muss überwunden werden. Diese Komplexität erfordert multiprofessionelle Teams, wie sie oft nur spezialisierte Institutionen bieten können. Im Rahmen der eigentlichen Psychotherapie sind die erprobten Behandlungskonzepte der Psychotraumatologie grundsätzlich effektiv. Ihnen sind allerdings aufgrund der hohen Komorbidität und der Belastung durch äußere Probleme Grenzen gesetzt. Deshalb haben ergänzende Behandlungsstrategien einige Bedeutung, vor allem solche, die zur Behandlung von sequenziell Traumatisierten und von Patienten mit strukturellen Störungen entwickelt wurden
\end{abstract}

DOI: https://doi.org/10.1007/s00278-005-0460-z

Posted at the Zurich Open Repository and Archive, University of Zurich

ZORA URL: https://doi.org/10.5167/uzh-156680

Journal Article

Published Version

Originally published at:

Maier, T (2006). Psychotherapie von Kriegs- und Folteropfern. Psychotherapeut, 51(1):47-54.

DOI: https://doi.org/10.1007/s00278-005-0460-z 
Psychotherapeut 2006 $51: 47-54$ DOI 10.1007/s00278-005-0460-z

Online publiziert: 10. Dezember 2005

(c) Springer Medizin Verlag 2005

\section{Redaktion}

U. Ehlert · Zürich

W. Schneider $\cdot$ Rostock

\section{Thomas Maier}

Ambulatorium für Folter- und Kriegsopfer (afk),

Psychiatrische Poliklinik des Universitätsspitals Zürich

\section{Psychotherapie von Kriegs- und Folteropfern}

\section{Zusammenfassung}

Im Zuge weltweiter Migrationsbewegungen kommen zunehmend Menschen zu uns, die durch Krieg-, Folter und Vertreibung traumatisiert sind. Die psychotherapeutische Versorgung dieser Menschen ist aus vielen Gründen hindernisreich und deshalb oft unzureichend. Neben eigentlichen psychischen Beschwerden liegen meist zusätzliche körperliche, soziale und aufenthaltsrechtliche Probleme vor. Die Sprachbarriere muss überwunden werden. Diese Komplexität erfordert multiprofessionelle Teams, wie sie oft nur spezialisierte Institutionen bieten können. Im Rahmen der eigentlichen Psychotherapie sind die erprobten Behandlungskonzepte der Psychotraumatologie grundsätzlich effektiv. Ihnen sind allerdings aufgrund der hohen Komorbidität und der Belastung durch äußere Probleme Grenzen gesetzt. Deshalb haben ergänzende Behandlungsstrategien einige Bedeutung, vor allem solche, die zur Behandlung von sequenziell Traumatisierten und von Patienten mit strukturellen Störungen entwickelt wurden.

\section{Schlüsselwörter}

Psychotherapie · Kriegs- und Folteropfer · Psychotraumatologie .

Sequenzielle Traumatisierung $\cdot$ Migration

\section{Psychotherapy of victims of war and torture}

\begin{abstract}
Within the context of worldwide migratory movements, numerous victims of war and torture are moving into western countries. Frequently, these patients are in need of psychotherapy, which is partly provided by specialized institutions. Psychotherapists of victims of war and torture have to deal with manifold additional physical, social, material, and legal problems of their clients. Therefore, such patients are best treated by multidisciplinary teams. The psychotherapy of such victims involves the classical methods for the treatment of PTSD; however, these methods may be of limited effect in these patients due to high comorbidity. Additional methods and concepts known from the treatment of complex PTSD and borderline personality disorders must be integrated into therapeutic strategies.
\end{abstract}

\section{Keywords}

Psychotherapy - Victims of war and torture - Psychotraumatology .

Complex traumatization · Migration 
- Migrationsbewegungen

\section{- "Überlebende organisierter Gewalt" \\ - Psychotraumatologie}

Die festgestellten Diagnosen gehen weit über die traumatypischen Hauptdiagnosen hinaus
Lernziel: Die Psychotherapie von Kriegs- und Folteropfern stellt an Therapeuten besondere Anforderungen, da neben posttraumatischen Symptomen im engeren Sinn in den meisten Fällen Komorbiditäten und zusätzliche psychosoziale Probleme vorhanden sind. Der vorliegende Text vermittelt einen Überblick über die institutionellen Voraussetzungen, die theoretischen Grundlagen und die therapeutischen Strategien im Zusamme nhang mit der Behandlung von Kriegs- und Folteropfern. Der Leser erkennt mögliche Fallen und Probleme, die sich bei der Therapie solcher Patienten ergeben können.

Studien zeigen, dass zumindest 10\% der Flüchtlinge in Industrieländern durch Krieg, Folter und Vertreibung in einem klinisch bedeutsamen Ausmaß psychisch (dazu oft auch physisch) traumatisiert sind (Fazel et al.2005). Die Behandlung dieser als Kriegs- und Folteropfer bezeichneten Menschen ist auf dem Hintergrund der weltweiten $\boldsymbol{\nabla}$ Migrationsbewegungen zu sehen und spielt sich nicht in einem geschützten Raum ab. Therapeuten sind - außer mit den medizinisch-psychotherapeutischen Problemen im engeren Sinne - gleichzeitig immer mit gesellschaftlichen, rechtlichen und ökonomischen Schwierigkeiten ihrer Patienten konfrontiert (Mollica et al.1987, Hauff u. Vaglum1995, Haenel2002). Obwohl heute weltweit über 200 spezialisierte Zentren für die Behandlung und Rehabilitation von Kriegs- und Folteropfern existieren, muss diese Patientengruppe als deutlich unterversorgt angesehen werden.

\section{Besonderheiten der Klientel}

Kriegs- und Folteropfer - einige ziehen Bezeichnungen wie \"Überlebende organisierter Gewalt" vor - stellen keine diagnostisch einheitliche Patientengruppe dar. Obwohl zumeist mit dem Oberbegriff $>$ Psychotraumatologie assoziiert, erfordert die Behandlung und Rehabilitation dieser Patienten den Einsatz zahlreicher weiterer Disziplinen des medizinisch-therapeutischen Systems, dazu Sozialarbeiter, Rechtsdienste, Laienhelfer, Religionsgemeinschaften, Pädagogen etc.

Die festgestellten Diagnosen gehen weit über die traumatypischen Hauptdiagnosen hinaus (Mollica1987, Steel et al.2002) und umfassen: Anpassungsstörungen, depressive, dissoziative und somatoforme Störungen, Abhängigkeitserkrankungen und Persönlichkeitspathologien. Die Patientengruppe der Kriegs- und Folteropfer ist also nicht durch umschriebene Diagnosen, sondern gerade durch eine Kombination von verschiedenen Symptomen und Syndromen gekennzeichnet, die sich auf dem Hintergrund der Traumatisierung entwickeln. An dieser Klientel zeigt sich besonders deutlich die Begrenzung von Reichweite und externer Validität der einschlägigen \ operationalisierten Diagnosen (Summerfield1999).

Krieg und Folter werden von vielen Autoren als besondere Formen der Traumatisierung angesehen, die sich nicht nur graduell, sondern qualitativ von "gewöhnlichen“ Traumata mit Todesgefahr (z. B. Unfällen, Naturkatastrophen, malignen Krankheiten) unterscheiden (Turner u. Gorst-Unsworth199o). Bei der $\triangleright$ Folter handelt es sich um eine absichtlich und ad hominem ausgeführte Grausamkeit, also um eine direkte Manifestation zwischenmenschlicher Gewalt. Solche Erfahrungen lösen weit mehr aus als Angstgefühle und „Überreizungen das Nervensystems“, sondern erschüttern elementare Überzeugungen und Sicherheiten, ja negieren die Möglichkeit und den Sinn menschlichen Zusammenlebens überhaupt. Folter ist eine der verstörendsten Erscheinungen der Zivilisation, zugleich eine universelle Konstante der Menschheitsgeschichte.

Die Auswirkungen der Folter auf den Menschen sind denn auch von einigen der bedeutendsten Autoren der Weltliteratur in ihren Werken behandelt worden, so von Dostojewskij, Kertész, Primo Levi, Sartre, Semprun, Tišma, Wiesel u. a. Insofern führt die Therapie von Kriegs- und Folteropfern weit über das Terrain der Psychotraumatologie hinaus, ja lässt im Grunde die Reduktion der Folgeerscheinungen auf ein medizinisch-psychotherapeutisches Problem inadäquat erscheinen (Graessner et al.1996). Dies dennoch zu tun, ist die paradoxe Prämisse der Behandlung. 
Kriegs- und Folteropfer, die in westlichen Ländern zur Behandlung kommen, durchlaufen praktisch ausnahmslos ein $>$ Asylverfahren. Ein sicherer $>$ Aufenthaltsstatus (z. B. als anerkannter Flüchtling) wird, wenn überhaupt, zumeist erst nach längerem Verfahren zuerkannt. Die betroffenen Personen haben aufgrund dieses provisorischen Rechtsstatus meist nur eingeschränkten Zugang zu voller medizinisch-psychotherapeutischer Versorgung. Neben rechtlichen und versicherungstechnischen Gründen (Haenel2002) behindern auch sprachliche und kulturelle Barrieren den Zugang zum Gesundheitswesen. Mehrere Problembereiche sind also charakteristisch für diese Klientel:

- Kulturelle Unterschiede und Missverständnisse von beiden Seiten können den Aufbau vertrauensvoller Beziehungen erschweren. Erwartungen an das Gesundheitswesen, an Ärzte und Therapeuten weichen oftmals sehr von landesüblichen Vorstellungen ab. Psychotherapie im westlichen Sinne ist vielen Migranten unbekannt, Konzepte und Begriffe von Patienten über Psyche, Emotionalität, Krankheit und Gesundheit können von den unsrigen sehr verschieden sein (Morris u. Silove1992).

- In der Regel reichen die gegenseitigen Fremdsprachenkenntnisse nicht aus, um sich in befriedigender Weise sprachlich zu verständigen. Der Einsatz von > Dolmetschern ist deshalb unumgänglich. Das hat den Vorteil, dass neben rein sprachlichen auch kulturelle, historische und religiöse Inhalte übersetzt werden können (Abdallah-Steinkopf1999). Die Rekrutierung von geeigneten, kompetenten und konstant abkömmlichen Dolmetschern ist allerdings schwierig und aufwendig, je nach erforderlicher Sprache manchmal unmöglich. Die Bezahlung der Dolmetscher wird größtenteils von den Kostenträgern nicht übernommen.

- Kriegs- und Folteropfer geben oft somatische und/oder somatoforme Beschwerden als Hauptsymptome an, da das Versorgungssystem darauf schneller und aktiver reagiert. In der Regel haben Opfer von Krieg und Folter tatsächlich verschiedene körperliche Probleme, die allerdings nur aus einem interdisziplinären Blickwinkel angemessen verstanden und behandelt werden können.

- Das laufende Asylverfahren beeinträchtigt eine Psychotherapie, da die Patienten sich nicht in Sicherheit fühlen bzw. tatsächlich nicht in Sicherheit sind. Außerdem hat der Gesundheitszustand von Asylbewerbern - teilweise vermeintlich, teilweise tatsächlich - einen Einfluss auf den Entscheid der Asylbehörden. In vielen Aufnahmeländern werden ärztliche Befunde als Indikatoren für die $>$ Glaubwürdigkeit der Angaben herangezogen, und vorhandene Gesundheitsstörungen können als Argumente für eine Aufnahme im Zielland geltend gemacht werden.

- Migranten werden als gesellschaftliche Gruppe beargwöhnt, häufig offen angefeindet und dienen als Projektionsfläche für verschiedene politische und soziale Konflikte. Sie leben in materiell knappen Verhältnissen und haben keine politische, soziale oder ökonomische Macht.

- Klinische Forschung, insbesondere $>$ Therapieforschung befasst sich bisher wenig mit der Gruppe der Kriegs- und Folteropfer (Ausnahmen z. B. Boehnlein et al.2004, Schauer et al.2005). Gründe dafür sind neben den fehlenden kommerziellen Interessen die Sprachbarriere und die Multimorbidität. Die methodischen Probleme, die daraus resultieren, werden in der Regel durch Ausschluss solcher Patienten umgangen.

\section{Institutionelle Voraussetzungen}

Wegen des geringen ökonomischen und sozialen Gewichts von Asylanten, vielleicht auch infolge einer latenten Aversion gegenüber Opfern im Allgemeinen (Reemtsma1999), ist das Interesse des öffentlichen Versorgungssystems an der Behandlung von Kriegsund Folteropfern gering. Die spezifische und umfassende sozialmedizinisch-psychotherapeutische Behandlung dieser Patienten findet daher in den vielen Ländern außerhalb der Regelversorgung statt, ein Umstand, über den bisher nur wenig Unbehagen zu spüren ist.

Viele spezialisierte Institutionen beruhen auf privaten Trägerschaften von Stiftungen, Vereinen und Hilfswerken, was bedeutet, dass nicht wenige Behandlungseinrichtungen
- Asylverfahren

- Aufenthaltsstatus

Dolmetscher

- Somatoforme Beschwerden

- Glaubwürdigkeit

Therapieforschung

- Öffentliches Versorgungssystem

Viele spezialisierte Institutionen beruhen auf privaten Trägerschaften und sind finanziell latent gefährdet 


\section{Spezialisierte Behandlungseinrichtungen}

Psychotherapie von Kriegs- und Folteropfern ist eine professionelle Leistung. Sie darf nicht als Freundschaftsdienst oder sozialpolitische Aktion missverstanden werden

Beim politischen Engagement und bei der Psychotherapie handelt es sich um zwei verschiedene Dinge mit verschiedenen Vorgehensweisen und verschiedenen Zielen

\section{- Burnout}

Die Psychotherapie von Kriegs- und Folteropfern unterscheidet sich nicht grundsätzlich von der Psychotherapie anderer Patientengruppen

\section{Spezifische Traumatherapien}

- PTSD latent gefährdet sind. Dadurch wiederholen diese Institutionen selbst das Schicksal ihrer Patienten: Sie haben einen unsicheren Status, können nicht langfristig planen, wissen nie, ob sie morgen noch weitermachen können. Aus einer solchen Situation heraus den Asylbewerbern Sicherheit und Zuversicht zu vermitteln, ist für Mitarbeiter bisweilen sehr schwierig. Es fehlt dann an der nötigen eigenen Sicherheit und Distanz, und die Gefahr undifferenzierter Identifikation wird verstärkt.

In manchen $>$ spezialisierten Behandlungseinrichtungen liegen die Gehälter unter dem Niveau vergleichbarer staatlicher Institutionen. Das hat Auswirkungen auf die Qualität der rekrutierbaren Fachkräfte, und manchmal kommen zu wenig breit ausgebildete Therapeuten oder „Quereinsteiger" aus anderen Berufsfeldern in dieses Metier. Auch ehrenamtliche Tätigkeit kommt vor, wogegen nicht grundsätzlich etwas einzuwenden ist. Allerdings müssen sich psychotherapeutisch Tätige der Konsequenzen und der daraus resultierenden Verzerrungen bewusst sein.

Die Patienten sollten wissen, dass ihre Behandlung eine professionelle Leistung ist, die Therapeuten sollen dafür entlohnt werden, und jemand muss für die Bezahlung aufkommen. Gerade wenn es um die Bearbeitung traumatischer Inhalte geht, brauchen Patient und Therapeut die Gewissheit, dass dies im Rahmen eines professionellen Settings geschieht und nichts mit reiner Betreuung oder Freundschaftsdiensten zu tun hat. Therapeuten sind keine selbstlosen „Gutmenschen“, die ohne Rücksicht auf eigene Bedürfnisse arbeiten. Dies wäre naiv und würde überdies bedeuten, die Patienten in ihrer Rolle als passive Hilfeempfänger zu bestätigen. Dennoch wird Psychotherapie von Kriegs- und Folteropfern von Außenstehenden (und manchmal auch von beteiligten Therapeuten selbst) als eine Art sozial-humanitäres Engagement verstanden und weniger als „normale“ therapeutische Tätigkeit. Dies entspringt unter anderem der Tradition der Anti-Folter-Bewegung, aus der heraus sich die Psychotherapie von Kriegs- und Folteropfern entwickelte und entsprechend als Fortsetzung bzw. als Bestandteil dieses Engagements aufgefasst wird (Somnier u. Genefke1986).

Der politische Kampf gegen Krieg und Folter ist in manchen Zentren eng mit der Behandlung der Opfer verknüpft. Psychotherapeuten sollten darauf achten, dass die individuellen Therapien nicht für diesen Kampf instrumentalisiert werden, denn es handelt sich beim politischen Engagement und bei der Psychotherapie um zwei verschiedene Dinge mit verschiedenen Vorgehensweisen und verschiedenen Zielen.

Auf Seiten der Helfer und Therapeuten gibt es also sehr verschiedene Motive, sich mit Kriegs- und Folteropfern zu befassen. Es darf nicht verschwiegen werden, dass auf diesem Weg auch eine Reihe von dysfunktionalen Einstellungen ins Spiel kommen, die sich auf die Behandlungsqualität und die dynamischen Abläufe innerhalb der Behandlungsteams auswirken. Nicht zufällig hatten und haben Institutionen, die sich mit Kriegs- und Folteropfern befassen, überdurchschnittlich häufig Probleme mit Teamkonflikten, hoher Personalfluktuation, > Burnout u. Ä. (Gurris2002). Die Behandlung von Kriegs- und Folteropfern soll wenn möglich nur im Rahmen von stabilen, regulären Strukturen stattfinden und von adäquat ausgebildeten Profis durchgeführt werden. Die Mitarbeiter sollen sich nicht selbst um die Finanzierung ihrer Institution bemühen müssen und nicht in Versuchung gebracht werden, sich als selbstlose Helden zu fühlen.

\section{Grundzüge der Psychotherapie}

Die Psychotherapie von Kriegs- und Folteropfern unterscheidet sich nicht grundsätzlich von der Psychotherapie anderer Patientengruppen. Insofern gibt es nicht die Psychotherapie von Kriegs- und Folteropfern. Zu empfehlen ist vielmehr die Kombination und Integration verschiedener Verfahren und Techniken (McIvor u. Turner1995, Turner u. GorstUnsworth1990).

Wichtige Impulse für die Behandlung von Kriegs- und Folteropfern kommen aus dem Bereich > spezifischer Traumatherapien, die auf dem Hintergrund der Einführung der - PTSD -Diagnose („post-traumatic stress disorder „, posttraumatische Belastungsstörung) ab 1980 entstanden sind und sich laufend weiter entwickeln (Ehlers u. Clark2ooo, Foa et al.200o, Gersons et al.200o). 
Dazu gehören verhaltenstherapeutisch orientierte Verfahren, die im Kern die therapeutische Exposition gegenüber dem traumatischen Erlebnis als wirksam ansehen (Schauer et al.2005). Auch $>$ EMDR („eye movement desensitization and reprocessing“) kann in diesem Kontext gesehen werden. Weiter werden integrative Ansätze beschrieben, Gruppentherapie und andere Verfahren wie psychodynamische Therapien, „testimony therapy“, „insight therapy“ u. a. (Übersicht bei Wilson u. Drožđek2004).

Da es sich bei Kriegs- und Foltererfahrungen und anschließender Flucht praktisch per definitionem um > sequenzielle Traumata handelt (Gorst-Unsworth u. Goldenberg1998, Mollica et al.1987), können neben eigentlichen posttraumatischen Symptomen (Wiedererleben, Angst, Vermeidung) auch somatoforme Störungen, Depression, chronische Suizidalität, selbstverletzendes Verhalten, Essstörungen, Substanzmissbrauch, emotionale Instabilität, dissoziative Zustände, Aggressivität u. a. auftreten. Die Behandlung orientiert sich primär an den vorliegenden Symptomen und bedient sich der Therapieverfahren, die sich in der Behandlung sequenziell Traumatisierter bewährt haben (Becker-Fischer u. Fischer1997). Zu berücksichtigen sind unbedingt die Wünsche und Vorstellungen der Patienten sowie die kulturelle Angemessenheit des gewählten Verfahrens (McIvor u. Turner1995, Morris u. Silove1992, Turner u. Gorst-Unsworth1990).

Neben variablen Techniken der Einzeltherapie haben sich auch $>$ Gruppentherapien und verschiedene nichtsprachliche Verfahren bewährt, da das rein sprachgebundene psychotherapeutische Einzelsetting oft nicht ideal ist. Dies ist auch der Fall, wenn soziale Probleme oder somatische Beschwerden für die Patienten im Vordergrund stehen. Die Involvierung von Sozialarbeiterinnen, Bewegungs- und Körpertherapeutinnen, Physiotherapeutinnen, somatischen Medizinerinnen etc. ist in sehr vielen Fällen sinnvoll, ja unumgänglich (Frey u. Valach1997, Gorst-Unsworth u. Goldenberg1998, Graessner et al.1996, Haenel2002, McIvor u. Turner1995).

Einige traumatisierte Migranten leiden stärker unter den aktuellen Lebensschwierigkeiten als unter früher erlebten Verletzungen (Gorst-Unsworth u. Goldenberg1998, Haenel2002, Hauff u. Vaglum1995). Interventionen auf sozialer, materieller oder rechtlicher Ebene tragen oft mehr zur Stabilisierung bei als rein psychotherapeutische Behandlung im engeren Sinne. Dieser Befund relativiert die Bedeutung der Psychotherapie gegenüber der Wichtigkeit von Sachhilfe und sozialer Unterstützung. Vor allem bei Patienten mit unsicherem Aufenthaltsstatus steht die äußere Realität gegenüber der inneren Realität des erlebten Traumas ganz im Vordergrund und muss vorrangig thematisiert werden.

Viele Therapeuten betonen zu Recht, dass bei der Behandlung von traumatisierten Migranten eine orthodox-abstinente Haltung fehl am Platz ist und klar zugunsten der Patienten Stellung bezogen werden muss. Damit ist gemeint, dass über die Unrechtmäßigkeit und die Ungeheuerlichkeit des Geschehenen und über die Verteilung von Schuld und Verantwortung kein Zweifel aufkommen soll. Es wäre hingegen falsch, sich völlig mit dem Schicksal der Patienten zu identifizieren und keine reflexive Distanz zu wahren. Allen muss klar sein, dass der Therapeut weder das Geschehene ungeschehen machen kann, noch verhindern kann, dass anderen Ähnliches widerfährt.

Auch sind Ungerechtigkeiten und Benachteiligungen im Aufnahmeland nicht aus der Welt zu schaffen, und selbst die Möglichkeit eines negativen Asylentscheides inklusive Rückschaffung ins Herkunftsland ist gegeben. Es hilft den Patienten nicht, wenn ihnen die Therapeuten vorführen, wie sie (die Therapeuten) Mühe haben, unangenehme äußere Realitäten zu akzeptieren. Gerade auf dem Hintergrund eines Übertragungsverständnisses ist eine professionelle therapeutische Haltung elementar, welche Begrenzungen aufgrund äußerer Gegebenheiten anerkennt.

Bei der Therapie (bzw. bei der diagnostischen Beurteilung) von Kriegs- und Folteropfern trifft man gelegentlich noch immer auf - Abwehrreflexe und Vorbehalte gegenüber der Anwendung psychopathologischer Begriffe (Somnier u. Genefke1986). Manche Therapeuten, aber auch nichttherapeutische Betreuer, Angehörige oder Patienten selbst, lehnen die Zuordnung zu pathologischen Kategorien ab, da sie darin eine Entwertung und Stigmatisierung erkennen wollen. Dahinter steckt auf Seiten der Patienten wohl der (verständliche) Wunsch, die Verletzung, die Beschädigung und die Erniedrigung un geschehen machen zu wollen. Auf Seiten der Therapeuten und Betreuer hingegen sind
- Exposition

- EMDR

Sequenzielle Traumata

Gruppentherapien

Einige traumatisierte Migranten leiden stärker unter den aktuellen Lebensschwierigkeiten als unter früher erlebten Verletzungen

In Bezug auf Menschenrechtsverletzungen und Kriegserlebnisse muss eine explizit parteiliche Haltung eingenommen werden. Abstinenz und Neutralität sind in diesem Punkt fehl am Platz

Eine professionelle therapeutische Haltung, die Begrenzungen aufgrund äußerer Gegebenheiten anerkennt, ist elementar

Abwehrreflexe 


\section{Anti-Folter-Bewegungen}

Es ist nicht sinnvoll, psychiatrische Diagnosen zu vermeiden, nur um Folteropfer als „, moralische Sieger" zu würdigen. Dabei würde eine offensichtliche Realität kollusiv verleugnet

Traumaexposition und -bearbeitung oder Stärkung der Abwehr und Stabilisierung der Verdrängung sollten nicht als Gegensatz, sondern als Ergänzungen verstanden werden

Exposition in sensu ist auch bei Kriegs- und Folteropfern theoretisch der Goldstandard der Behandlung

Sicherheit ist die erste und wichtigste Voraussetzungfür jede Art von psychotraumatologischer Behandlung

Die Verunsicherung ist eine äußere Realität

Dissoziative Zustände ebenfalls verleugnende oder allenfalls ideologische Gründe ausschlaggebend, indem auf dem Hintergrund von $>$ Anti-Folter-Bewegungen psychopathologische Begriffe für Klienten abgelehnt werden.

Die Ansicht, es handle sich bei Kriegs- und Folteropfern nicht um Patienten, sondern um Gesunde, denen bloß Schlimmes widerfahren ist, verfehlt und verharmlost das Problem. Schließlich haben diese Menschen offensichtliche Beschwerden und verspüren einen Leidensdruck (sonst kämen sie nicht in Behandlung). Es ist ein „double bind“, einerseits die Notwendigkeit einer Behandlung zu betonen, anderseits aber zu versichern, es liege eine „normale“ Reaktion auf abnormale Umstände vor. Im Gegenzug wird gegenüber Kostenträgern und im Rahmen des Asylverfahrens oft recht freizügig mit dem Krankheitsbegriff operiert, dies auch im Interesse und mit dem Einverständnis der Patienten.

\section{Traumaexposition oder Stärkung der Abwehr?}

Quer durch interdisziplinäre Behandlungskonzepte zieht sich die Frage, ob Traumaexposition und -bearbeitung oder im Gegenteil Stärkung der Abwehr und Stabilisierung der Verdrängung vorzuziehen ist. Der Gegensatz ist in dieser Akzentuierung natürlich unsinnig, da es nicht um ein Entweder-oder geht, sondern vielmehr um die Frage, wieviel, wovon und zu welchem Zeitpunkt.

Der aktuelle Stand des Wissens spricht dafür, dass bei der psychotherapeutischen Behandlung von Traumafolgen die Exposition, also das möglichst ausführliche und komplette Durcharbeiten der traumatischen Erfahrung, erfolgversprechend ist (Foa et al.200o). Daraus kann man extrapolieren, dass auch bei der Behandlung sequenziell Traumatisierter (inkusive Kriegs- und Folteropfern) grundsätzlich die Exposition in sensu gegenüber den traumatischen Erlebnissen als theoretischer Goldstandard angesehen werden kann. Zugleich wird klar, dass einem solchen Vorgehen auf praktischer Ebene mannigfaltige Hindernisse im Wege stehen und es demzufolge eher um die Frage geht, in welchen Fällen, zu welchem Zeitpunkt in welcher Weise exponiert werden soll und in welchen Fällen dies eher zu unterlassen ist.

Die psychotherapeutische Behandlung von traumatisierten Menschen muss berücksichtigen, dass die Verarbeitung eines Traumas (auch ohne Therapie) in Stadien abläuft. Bekanntlich ist die erste und wichtigste Maßnahme nach einem Trauma die Gewährleistung von Sicherheit. Das psychische Trauma besteht in seiner Essenz gerade und hauptsächlich darin, dass ein grundsätzliches Gefühl von Sicherheit und Unantastbarkeit abrupt zerstört, zerschlagen, ja als Illusion und Unmöglichkeit entlarvt wurde.

Die Wiederherstellung des Gefühls der Sicherheit ist daher zentral, gleichzeitig aber im Falle von Kriegs- und Folteropfern praktisch unmöglich. Die Flucht aus der Heimat, die Vertreibung, das Herausgerissenwerden aus dem Familienverband und aus der sozialen Gruppe verstärken im Gegenteil die Verunsicherung, ja sind per se Traumatisierungen (Gorst-Unsworth u. Goldenberg1998, Hauff u. Vaglum1995, Mollica et al.1987). Die Lebensbedingungen im Zielland, namentlich die drohende Rückschaffung, verhindern in der Regel nachhaltig, dass sich ein Gefühl der Sicherheit und der Unantastbarkeit wieder einstellen kann. Therapeuten müssen sich bewusst sein, dass Asylbewerber per definitionem nicht in Sicherheit sind. Die Verunsicherung ist nicht ein psychotherapierbares inneres Gefühl, sondern eine äußere Realität. Eine Reihe weiterer Begrenzungen bzw. Kontraindikationen gegenüber Expositionsbehandlungen sind zu beachten:

Das Auftreten von > dissoziativen Zuständen ist nach Extremtraumatisierungen häufig und kann als Ausdruck noch nicht wiedergewonnener, minimaler Sicherheit angesehen werden. Diese Patienten fühlen sich noch zu stark von den traumatischen Erinnerungen überschwemmt und belastet, sodass sie sich nur mit dem Mechanismus der Dissoziation davor schützen können. Hier muss zuerst an der Stabilisierung gearbeitet werden, bevor an eine Exposition gedacht werden kann. Als weiterer Ausdruck fehlender Grundstabilität kann anhaltendes Agieren außerhalb der Therapie in Form von wiederholten somatischen Notfallbehandlungen, > Suizidalität, Suchtmittelkonsum, Impulsdurchbrüchen etc. gesehen werden. Auch hier ist die Arbeit an der Stabilität, der inneren Sicherheit, der Ich-Kontrolle essenziell, bevor man an die Bearbeitung traumatischer Inhalte gehen kann. 
Ebenfalls sollten eine allgemein schlechte soziale Integration und dominierende äußere Probleme prioritär angegangen werden, bevor man eine Exposition gegenüber traumatischem Material anstrebt. Die Verbesserung der allgemeinen Lebensumstände, auch z. B. das Erlernen der Landessprache, verbessert oft die Befindlichkeit und das Selbstvertrauen so stark, dass die normalen Verarbeitungsmechanismen greifen und später weitere Behandlungen überflüssig werden können. Denn am anderen Ende der Skala soll auch ein sehr gutes psychisches und soziales Funktionieren als relative Kontraindikation für Traumaexposition genannt werden. Schließlich sollen resiliente Personen nicht unnötig von übereifrigen Therapeuten verunsichert werden.

Da Folter und Krieg für einheimisches Gesundheitspersonal (zum Glück) meist sehr fremde und schwer vorstellbare Geschehnisse sind, fällt das Sprechen über extreme Traumata den Behandelnden manchmal schwerer als den Betroffenen selbst. Die Angst vor eigenen heftigen Affekten wie Entsetzen, Ekel, Ohnmacht, Wut etc. können bei Therapeuten ein Gefühl der Unsicherheit und Überforderung aufkommen lassen (Zurek u. Fischer2003). In der Folge wird das Ansprechen traumatischer Erlebnisse vermieden, wobei es zu einem gemeinsamen (kollusiven) Vermeiden kommen kann, da es auch den Patienten recht ist, nicht daran zu rühren.

Ein Gefühl der Überforderung ist von Therapeuten selbst auf jeden Fall ernst zu nehmen und soll nicht einfach als eigene Insuffizienz abgehakt werden. Das Problem der - Traumatisierung der Therapeuten durch die Patienten („vicarious traumatization“) gehört zu den besonderen Herausforderungen des Metiers. Die eigene Belastbarkeit muss als Maß und Begrenzung in jeder Form von Psychotherapie den äußeren Rahmen bilden. Dies ist ein Teil des Settings und damit ein wichtiges Element der Behandlung.

Andererseits können Therapeuten durch eine forcierte und invasive Exploration traumatischer Erlebnisse auch Schaden anrichten. Entgegen einer offenbar in Therapeutenkreisen anzutreffenden Meinung, ist das exzessive und wiederholte Schildern möglichst schlimmer Erfahrungen nicht automatisch heilsam und immer nur von Gutem. Das Erzählen und Repetieren von grausamen Erlebnissen kann retraumatisierend sein, und es können sich überdies eine Reihe dysfunktionaler Momente darin ausdrücken ${ }^{1}$. Diese zu erkennen und allenfalls bremsend oder unterbrechend zu intervenieren, ist die Aufgabe des Therapeuten.

Beispielsweise kann die Schilderung schlimmer Gewalterfahrungen Elemente sadomasochistischer Reinszenierungen enthalten, wobei die Rollenverteilung zwischen Patient und Therapeut unterschiedlich ist. Manche Patienten quälen ihre Therapeuten mit exzessiven Schilderungen von Folterszenen und wiederholen so die Foltersituation mit umgekehrten Vorzeichen. Extrem traumatisierte Patienten können Übertragungskonstellationen herbeiführen, die sich nicht unterscheiden von sadistischen, narzisstischen oder paranoiden Übertragungen anderer Patientenkategorien. Die Reinszenierung von Gewalt, Aggression und Destruktivität gehört zum notwendigen therapeutischen Prozess (Zurek u. Fischer2003), wobei bei Kriegs- und Folteropfern häufig eher unterschwelliges und indirektes Ausagieren zu beobachten ist. Dieses wiederholt spezifische Aspekte der konkreten traumatischen Erfahrung.

Man darf aber nicht vergessen, dass diese Menschen zumeist eine normale prätraumatische Sozialisation durchlaufen haben und deshalb in der Regel parallel oder zumindest im Längsschnitt auf $>$ reife Ich-Strukturen zurückgreifen können.

\section{Fazit für die Praxis}

Die psychotherapeutische Behandlung von Kriegs- und Folteropfern ist aus verschiedenen Gründen anspruchsvoll: Die Patienten sind in der Regel komplex traumatisiert, leiden meist an multiplen psychischen, somatischen und psychosomatischen Beschwerden, sind aufgrund ihres Migrantenschicksals sozial und kulturell entwurzelt und sprechen unsere Sprache nicht. Es geht also nicht nur um psychotraumatologisch besonders komplizierte

${ }^{1}$ Wir besprechen hier nur dysfunktionale Mechanismen auf Seiten der Patienten. Solche können auch auf Seiten der Therapeuten eine Rolle spielen.
Die Stabilisierung der sozialen Situation ist einer der wichtigsten Faktoren für die Verbesserung der allgemeinen psychischen Befindlichkeit

Das Sprechen über extreme Traumata fällt den Behandelnden manchmal schwerer als den Betroffenen selbst

\section{Traumatisierung der Therapeuten}

Therapeuten können durch eine forcierte und invasive Exploration traumatischer Erlebnisse auch Schaden anrichten

\section{Sadomasochistische} Reinszenierungen 
Behandlungen, sondern darüber hinaus um transkulturelle Schwierigkeiten und um sozialpsychiatrische Probleme. Für die Psychotherapie im engeren Sinne sind neben den klassischen psychotraumatologischen Therapieformen Modelle, Konzepte und Einstellungen wegweisend, wie sie in der Behandlung von strukturell gestörten Patienten angewandt werden. Klarheit der Rolle und des Settings, Begrenzung des Agierens und funktionierende interdisziplinäre Teams sind Stichworte, die angemessene Behandlungskonzepte charakterisieren.

\section{Korrespondierender Autor \\ Dr. Thomas Maier}

Ambulatorium für Folter- und Kriegsopfer (afk), Psychiatrische Poliklinik des Universitätsspitals Zürich, Culmannstraße 8, CH-8091 Zürich

E-Mail: thomas.maier@usz.ch

Interessenkonflikt: Es besteht kein Interessenkonflikt. Der korrespondierende Autor versichert, dass keine Verbindungen mit einer Firma, deren Produkt in dem Artikel genannt ist, oder einer Firma, die ein Konkurrenzprodukt vertreibt, bestehen. Die Präsentation des Themas ist unabhängig und die Darstellung der Inhalte produktneutral.

\section{Literatur}

Abdallah-Steinkopf B (1999) Psychotherapie bei Posttraumatischer Belastungsstörung unter Mitwirkung von Dolmetschern. Verhaltenstherapie 9: 211-220

Becker-Fischer M, Fischer G (1997) Folgetherapie nach sexuellem Missbrauch in Psychotherapie und Psychiatrie. In: Egle U (Hrsg) Sexueller Missbrauch, Misshandlung, Vernachlässigung: Erkennung und Behandlung psychischer und psychosomatischer Folgen früher Traumatisierung. Schattauer, Stuttgart

Boehnlein JK, Kinzie D, Sekiya U, Riley C, Pou K, Rosborough $B$ (2004) A ten-year treatment outcome study of traumatized Cambodian refugees. J Nerv Ment Dis 192: 658-663

Ehlers A, Clark DM (2000) A cognitive model of posttraumatic stress disorder. Behav Res Ther 2000 38(4): 319-345

Fazel M, Wheeler J, Danesh J (2005) Prevalence of serious mental disorder in 7000 refugees resettled in western countries: a systematic review. Lancet 365: 1309-1314

Foa EB, Keane TM, Friedman MJ (2000) Effective treatments for PTSD. Practice guidelines from the International Society for Traumatic Stress Studies. Guilford Press, New York

Frey C, Valach L (1997) The Swiss Red Cross Therapy Centre for Torture Victims in Berne, Switzerland. Torture 7(1): 21-23

Gersons B, Carlier IV, Lamberts RD, van der Kolk BA (2000) Randomized clinical trial of brief eclectic psychotherapy for police officers with posttraumatic stress disorder. J Trauma Stress 13(2): 333-347

Gorst-Unsworth C, Goldenberg E (1998) Psychological sequelae of torture and organised violence suffered by refugees from Iraq. Trauma-related factors compared with social factors in exile. Br J Psychiatry 172: 90-94

Graessner S, Gurris N, Pross C (Hrsg) (1996) Folter. An der Seite der Überlebenden. Unterstützung und Therapien. Beck'sche Reihe 1183, München

Gurris NF (2002) Überlegungen zur stellvertretenden Traumatisierung bei Therapeuten in der Behandlung von Folterüberlebenden. Psychotraumatologie 45: http://www.thieme-connect.com/ejournals/abstract/psychotrauma/doi/10.1055/s-200235265
Haenel F (2002) Psychisch reaktive Kriegs- und Folterfolgen. Die Einflüsse sozialer und rechtlicher Umstände auf die psychotherapeutische Behandlung von Folterüberlebenden. Psychotherapeut 47: 185-188

Hauff E, Vaglum P (1995) Organised violence and the stress of exile. Predictors of mental health in a community cohort of Vietnamese refugees three years after resettlement. Br J Psychiatry 166: 360-367

Mclvor RJ, Turner SW (1995) Assessment and treatment approaches for survivors of torture. $\mathrm{Br} \mathrm{J}$ Psychiatry 166: 705-711

Mollica RF, Wyshak G, Lavelle J (1987) The psychological impact of war trauma and torture on Southeast Asian refugees. Am J Psychiatry 144: 1567-1572

Morris P, Silove D (1992) Cultural influences in psychotherapy with refugee survivors of torture and trauma. Hosp Commun Psychiatry 43: 820-824

Reemtsma JP (1999) „Trauma“ - Aspekte der ambivalenten Karriere eines Konzepts. Persönlichkeitsstörungen 4: 207-214

Schauer M, Neuner F, Elbert T (2005) Narrative exposure therapy. A short-term intervention for trauamtic stress disorders after war, terror, or torture. Hogrefe \& Huber, Göttingen

Somnier FE, Genefke IK (1986) Psychotherapy for victims of torture. Br J Psychiatry 149: 323-329

Steel Z, Silove D, Phan T, Bauman A (2002) Long-term effect of psychological trauma on the mental health of Vietnamese refugees resettled in Australia: a population-based study. Lancet 360(9339): 1056-1062

Summerfield D (1999) A critique of seven assumptions behind psychological trauma programmes in waraffected areas. Soc Sci Med 48: 1449-1462

Turner SW, Gorst-Unsworth C (1990) Psychological sequelae of torture. A descriptive model. Br J Psychiatry 157: 475-480

Wilson JP, Drožđek B (eds) (2004) Broken spirits. The treatment of traumatized asylum seekers, refugees, war and torture victims. Brunner-Routledge, New York Hove

Zurek G, Fischer G (2003) Übertragung und Gegenübertragung in der Psychotherapie von Patienten mit psychotraumatischen Belastungssyndromen (PTBS). ZPPM 1: 7-17 